\title{
Enseignement universitaire: ne pas croire à un seul modèle
}

\author{
L'Université de demain doit être différente, plus libre de ses choix
}

\section{J. Martin}

En octobre 2005, j'ai visité l’Université de Princeton, dans le New Jersey (celle où Albert Einstein a enseigné durant plusieurs décennies), membre du groupe prestigieux de Hautes Ecoles américaines dénommé Ivy League. Impressionnant. Sentiment de découvrir les voies et moyens de la création d'une élite intellectuelle peu ordinaire. Je n'insiste pas sur le site, architecture et allure typiques des universités anglo-saxonnes classiques. Avec des indications fortes qu'on est encore devant une communauté universitaire. Par la taille d'abord: 4600 étudiants undergraduate (jusqu'au master) et 1400 doctorants (graduate students). Six mille au total pour une institution classée récemment au $7^{\mathrm{e}}$ rang mondial des universités; alors que celle de Lausanne en a plus de 10000 aujourd'hui, que de nombreuses universités européennes en ont des dizaines de milliers, celle de Rome 180 000. Manifestement, à Princeton, on veut rester petit ... et continuer à exceller. Plus de $95 \%$ des étudiants vivent sur le campus: les études et la vie estudiantine extrascolaire sont d'une telle intensité qu'on est motivé à ne pas s'en éloigner.

La petite taille fait qu'on peut privilégier le cousu main, de diverses manières. L'étudiante qui nous guide signale qu'une exigence académique est de se familiariser avec une langue étrangère. Que se passe-t-il, a-t-il été demandé, si l'étudiant(e) souhaite travailler un idiome exotique et peu connu? Réponse: l'université cherchera à l'extérieur un enseignant adéquat et le paiera pour vous instruire ... Et de citer d'autres exemples du même genre.

Il y a très peu de cours obligatoires, on encourage l'étudiant à formater lui-même son programme. De manière compréhensible dans une petite institution avec un ratio élevé de personnel enseignant par étudiant, un encadrement substantiel peut être offert de routine, les étudiants ne peinent pas à solliciter du temps de leurs maîtres. Tous les professeurs, aussi éminents et suroccupés soient-ils, donnent un enseignement en classe.

Bien sûr, il faut rappeler que Princeton et d'autres universités fameuses sont privées, que leurs anciens élèves (alumni) forment des clubs aisés et généreux vis-à-vis de leur Alma Mater sans compter les fondations philanthropiques et autres mécènes. Qu'il y a une sélection serrée des étudiants et que tout cela n'est pas très «social», au sens de l'accès potentiellement universel à l'enseignement supérieur. Cependant, on relèvera que $55 \%$ des étudiants de Princeton bénéficient d'une aide financière de la part de l'institution, tous ne sont donc pas issus des milieux historiquement ou économiquement prestigieux - même si dans certaines familles des générations successives passent par la même université de la Ivy League. Mais il y a aussi un effort déterminé d'admettre en nombre des jeunes brillants d'origine modeste et de minorités. A noter: alors que, en général aux Etats-Unis, il s'agit de prêts payables en retour quand le professionnel issu de l'Ecole est parvenu à bonne fortune, Princeton est une des seules institutions dont les aides financières sont non remboursables (grants).

Il n'est pas imaginable, à l'évidence, que tous les jeunes atteignant le niveau tertiaire bénéficient de conditions de ce type. Il faut une institution ou un environnement bien riches pour les réaliser - et nous voulons en Europe un accès large à l'enseignement supérieur. Toutefois, cette visite a renforcé chez moi l'idée discutée actuellement que, dans un monde de ressources toujours limitées et où quoiqu'on fasse certains sont plus talentueux que d'autres, il convient d'admettre l'émergence de plusieurs types de Hautes Ecoles. Je connais bien le discours sur l'enseignement universitaire qui doit être, précisément, universel et de qualité dans tous les domaines. Cet idéal ne peut plus, aujourd'hui, être concrétisé dans toutes ses conséquences. Dans un petit pays comme le nôtre par exemple, avec un grand nombre de Hautes Ecoles, il est impératif que ces dernières, en se concertant, décident de priorités, renoncent à rechercher l'inatteignable universalité (sauf à admettre de le faire dans la médiocrité); je n'ignore pas qu'elles ont commencé. On sait que la question se pose très concrètement pour la médecine. Si les soins doivent rester autant que faire se peut proches de la population, 
la recherche de haut niveau demande des centres suffisamment étoffés (et par conséquent attractifs), qui ne peuvent pas être multipliés.

Le magazine The Economist a publié le 10 septembre 2005 une enquête sur le monde universitaire, sous le titre «The brains business». Ce dossier a l'air d'une ode à l'université anglo-saxonne et particulièrement américaine, mais il importe ici de dépasser un réflexe anti-Etats-Unis. Il insiste spécialement sur l'importance pour les universités de diversifier leur financement, de rechercher des collaborations avec le secteur privé, y compris l'industrie (tout en étant attentif aux conflits d'intérêt et aux risques de perte d'indépendance), et de bénéficier d'une large liberté d'action par rapport aux pouvoirs publics. Quatre raisons aux mutations indispensables: la rapide augmentation du nombre d'étudiants («massification»sic), l'émergence de l'économie du savoir comme secteur important, la mondialisation, et la compétition entre universités, qui se marque de plus en plus au niveau international. The Economist estime, de façon catégorique, que les systèmes vivant essentiellement de l'argent public - et sous contraintes publiques (France, Allemagne, Suisse aussi) ne peuvent d'ores et déjà plus soutenir la comparaison avec des institutions bénéficiant d'une plus grande autonomie - et que ce handicap ira en s'aggravant. Avec en passant une pique logique à la situation des chercheurs «de carrière», fonctionnaires, que connaissent nos voisins français.

S'agissant d'autonomie, la nouvelle loi sur l'Université de Lausanne de juillet 2004 donne à cette dernière une liberté qu'elle n'avait pas et qui peut lui permettre de faire preuve de dynamisme et d'innovation. Encore faudra-t-il que son parlement (Conseil de l'Université), ou un peu plus loin le Parlement cantonal (comme on l'a vu récemment à Neuchâtel) ne bloquent pas des initiatives dans le sens de la souplesse, de la détermination de priorités (et donc de certains abandons), et de la recherche d'argent privé, au motif qu'on s'éloignerait de l'Université universelle, démocratique, républicaine, égale pour tous ...

Le propos ici n'est pas de s'opposer au fait que plus de jeunes aient accès au niveau tertiaire d'études. Il est de rompre une lance en faveur d'une évolution des doctrines et des pratiques: si on entend rester excellent, dans un monde de ressources toujours limitées une fois encore, il faut pouvoir sélectionner: les champs d'étude et de recherche, les étudiants aussi, les manières dont on investit les moyens disponibles. Etablir des niveaux différents, aller vers plusieurs types d'institutions tertiaires. Nous connaissons en Suisse un développement intéressant dans ce sens, les HES (Hautes Ecoles Spécialisées), malgré le fait qu'ici et là on semble mettre en place des usines à gaz bureaucratiques - souhaitons que ce ne soit que maladie de jeunesse. En ce qui concerne les Universités au sens strict, on doit mettre sur pied conjointement des centres particulièrement performants susceptibles de rester dans le peloton de tête dans leur domaine au niveau mondial; d'autres mettant un accent plus important sur la préparation à la vie professionnelle et moins à la recherche (seule une centaine des 3200 institutions d'enseignement supérieur des Etats-Unis sont de véritables research universities - mais rien n'empêche de passer des unes aux autres si on en a les compétences).

Il s'agit selon The Economist de «créer un système d'enseignement supérieur qui ménage un espace pour un certain nombre de elite universities tout en instruisant aussi les grands nombres d'étudiants moyens». Deux conclusions majeures, en termes bruts: primo, diversifier les sources de revenus des universités et, segundo, dans une métaphore à la chinoise, laisser fleurir mille fleurs académiques (que chaque institution trouve sa vocation propre, et donc son public propre - les étudiants). Plus facile à dire qu'à faire en Europe continentale. Et certains crieront au scandale devant de telles perspectives, à l'abandon du modèle qui nous a bien servis aux $19^{\mathrm{e}}$ et $20^{\mathrm{e}}$ siècles. Mais comment ne pas voir que c'est inéluctable? On ne peut pas vouloir plaquer les principes qui valent à juste titre pour la scolarité obligatoire jusqu'au stade de l'obtention d'un doctorat ... Ce qui veut dire entre autres qu'il n'est pas inadmissible de prélever des taxes d'étude qui ne soient pas simplement symboliques (des dispositifs adéquats d'aide financière à ceux qui en ont besoin étant mis en place).

En conclusion, il ne s'agit pas de se calquer sans discernement sur un modèle américain, tout en sachant cependant que les grands pays émergents s'en inspirent - Chine, Inde, dont il est clair qu'ils deviennent des concurrents formidables. Mais il faut, en matière d'enseignement et de recherche universitaires de haut niveau, prendre la mesure d'une évolution internationale dont la logique n'est pour l'essentiel, objectivement, guère contestable; ceci sur le décor des contraintes d'un petit pays et du fédéralisme qui le caractérise. Il importe de gérer - et adapter - les «vaches sacrées» dont certains jugent encore qu'elles sont intouchables au risque de perdre notre âme. Comme dans d'autres domaines, la Suisse a un intérêt éminent à réaliser qu'autour d'elle tout change vite et que, plus tôt que nous ne l'imaginons, nous pourrions nous retrouver à la traîne. 